\title{
The impact of estimated glomerular filtration rate equations on chronic kidney disease staging in pediatric renal or heart transplant recipients
}

\author{
Aram Ben Vroling ${ }^{1}$ Eiske Margaretha Dorresteijn ${ }^{2}$. \\ Karlien Cransberg ${ }^{2} \cdot$ Yolanda Brigitta de Rijke $^{1}$
}

Received: 5 June 2015 /Revised: 27 November 2015 / Accepted: 4 January 2016 / Published online: 9 February 2016

(C) The Author(s) 2016. This article is published with open access at Springerlink.com

\begin{abstract}
Background The aim of this study was to evaluate the performance of selected pediatric estimated glomerular filtration rate (eGFR) equations in relation to the clinical management of children after renal or heart transplantation or postchemotherapy treatment.

Methods This study was a retrospective cross-sectional analysis of 61 children whose glomerular function (GFR) had been determined using a single-dose inulin clearance (iGFR) method. Eight equations for estimating the GFR were evaluated for bias, agreement, accuracy, and clinical stratification. Results The outcome of all eight eGFR equations differed from the value determined using the iGFR method, with the mean bias ranging from -3.4 to $20.7 \mathrm{ml} / \mathrm{min} / 1.73 \mathrm{~m}^{2}$ and $90 \%$ accuracy ranging from 16 to $26 \%$. All eGFR equations overestimated renal function in patients with decreased kidney function as determined by the iGFR method and underestimated renal function in patients with normal kidney function. Consequently, based on the eGFR values, patients with low GFR values according to the iGFR method were staged in a less severe chronic kidney disease (CKD) category, and patients with normal GFR values according to the iGFR method were staged in a more severe CKD category. The
\end{abstract}

Aram Ben Vroling and Eiske Margaretha Dorresteijn contributed equally to this work.

Eiske Margaretha Dorresteijn

e.dorresteijn@erasmusmc.nl

1 Department of Clinical Chemistry, Erasmus University Medical Center, Rotterdam, The Netherlands

2 Department of Pediatric Nephrology, Erasmus University Medical Center-Sophia Children's Hospital, Wytemaweg 80, 3015 CN Rotterdam, The Netherlands percentage of correctly classified patients ranged from 32.6 to $41.6 \%$.

Conclusions In our cohort we found the CKiDIII equation to be the best alternative to calculating the GFR using the inulin clearance method, closely followed by the Hoste and the revised Grubb equations. The performances of all eight eGFR equations assessed were moderate at best and only slightly better than the easy-to-do bedside Schwartz equation.

Keywords Children - Estimated glomerular filtration rate . Chronic kidney disease $\cdot$ Renal transplantation $\cdot$ Heart transplantation $\cdot$ Chemotherapy $\cdot$ Schwartz equation

\section{Introduction}

The ideal reference method for defining glomerular filtration rate (GFR) is measuring the clearance of a marker that is freely filtered in the glomerulus, not metabolized, and not secreted by or re-absorbed in the tubule. The gold standard for calculating the GFR is based on measuring blood levels or urine excretion following a single injection or a steady state infusion of an exogenous marker, such as iohexol, iothalamate, inulin, or ${ }^{51} \mathrm{Cr}$-EDTA, from which the GFR can be calculated $[1,2]$. However, these tests are burdensome and expensive, and thus less feasible for routine clinical practice [3]. Consequently, clinicians prefer the alternative, endogenous markers, mostly with the use of serum creatinine-based formulas, validated against reference methods. There are, however, a number of limitations associated with creatinine-based methods, such as the creatinine level being related to muscle mass, creatinine being actively secreted by the proximal tubule in the case of severe renal functional impairment, and creatinine being metabolized extrarenally by intestinal bacteria [4-8]. 
This has led to growing interest in alternative methods to calculate the GFR, including the use of cystatin C, which was introduced as a marker for glomerular filtration in 1985 [9]. Cystatin $\mathrm{C}$ is a $13.3-\mathrm{kDa}$ molecule that is ubiquitously produced by all nucleated cells, freely filtered in the glomerulus, and subsequently completely re-absorbed and degraded in the proximal tubule [10]. The cystatin $\mathrm{C}$ production rate per cell is relatively stable throughout life from 2 years of age, and it thereby may be an attractive marker of GFR in both children and adults $[10,11]$. It has been described as an almost ideal marker, with only few limitations, such as its extrarenal elimination (though presumed to be negligible), its dosedependent correlation to the use of glucocorticoids, and the influence of thyroid dysfunction or diabetes mellitus [12].

The considerable body of research which has focused on the development and validation of equations for estimating GFR using creatinine, cystatin $\mathrm{C}$, or a combination of both [13-23] has mainly involved cohorts with specific ranges of age and kidney function. As a consequence, the outcomes of these equations are hard to extrapolate to other populations.

In the study reported here, , we retrospectively compared the performance of eight selected equations used to estimate GFR, all published between 1999 and 2014 and based on serum creatinine and/or cystatin C, with the GFR calculated from a single-injection inulin clearance method, in a cohort of children and adolescents after heart or kidney transplantation, or post nephrotoxic treatment of a malignancy. We also assessed the reliability of the different equations to predict chronic kidney disease (CKD) classification and thereby the clinical management of the individual patient.

\section{Materials and methods}

\section{Patients and study design}

This study is a retrospective, cross-sectional analysis of patients in whom the GFR was assessed by a single-dose inulin clearance method (iGFR). In our tertiary pediatric nephrology center, inulin clearance is a routine test for patients after renal transplant, heart transplant or post nephrotoxic chemotherapy. In renal transplant patients, iGFR is performed yearly in the first 3 years after transplantation and every other year thereafter. In heart transplant patients, it is performed 1 year after heart transplantation and in some cases pre-heart transplantation, while in some oncology patients it is performed at least 1 year after cessation of chemotherapy. Our patient cohort consisted of only patients in whom serum creatinine, urea, and cystatin $\mathrm{C}$ were measured concomitantly.

We first performed a PubMed literature search with the MESH terms "infant", "child", "adolescent", "glomerular filtration rate", "creatinine", and "cystatin C", initially selecting cystatin $\mathrm{C}$ - and/or creatinine-based GFR equations which had been published during the last 20 years and developed in a pediatric cohort. We then selected the equations in those studies that matched ours in terms of cohort age, range of GFR, and biochemical analysis used. The criteria were: (1) creatinine measurement by enzymatic assay, (2) cystatin $\mathrm{C}$ measurement by turbidimetric assay, and (3) the equation had to be developed for use in a pediatric and adolescent cohort with a range of renal function from severely decreased to normal. Studies originally designed for adult cohorts, but whose application was analyzed in pediatric patients, were also included.

\section{Biochemical analysis}

All biochemical analyses were performed on a Hitachi analyzer (Roche Diagnostics GmbH, Penzberg, Germany). Creatinine was measured using the Creatinine Plus version 2 enzymatic assay (Roche Diagnostics), with interassay coefficients of variation $(\mathrm{CV})$ of 1.0 and $0.8 \%$ at 85.7 and $370 \mu \mathrm{mol} / \mathrm{l}$, respectively. Blood urea was measured using the UREAL assay (Roche Diagnostics), with interassay CV of 2.2 and $1.3 \%$ at 6.88 and $23.7 \mu \mathrm{mol} / 1$, respectively. Cystatin $\mathrm{C}$ was measured using the Tina-quant cystatin $\mathrm{C}$ assay (Roche Diagnostics), with interassay CV of 1.9 and $2.5 \%$ at 1.08 and $4.61 \mathrm{mg} / 1$, respectively. The inulin analysis and subsequent GFR calculation were performed as described by van Rossum et al. [24] with blood sampling at 10, 30, 90, and $240 \mathrm{~min}$ after injection of $5000 \mathrm{mg} / 1.73 \mathrm{~m}^{2}$ polyfructosan (Inutest $25 \%$; Fresenius, Linz, Austria). All patients had a single cannula placed for the inulin (polyfructosan) injection, which was thoroughly flushed prior to blood sampling. The inulin clearance was calculated using MW/Pharm 3.5 (Mediware, Groningen, the Netherlands), a pharmacokinetic computer program using a two-compartment model by Bayesian analysis to calculate estimated GFR (eGFR). This Bayesian analysis combines information from the population pharmacokinetic parameters with information derived from the actual individual concentrations of samples to estimate the individual pharmacokinetic parameters. The calculation is based on the following pharmacokinetic parameters: plasma clearance of inulin, volume of distribution of the central compartment, intercompartmental clearance, volume of distribution of the peripheral compartment. Data on these pharmacokinetic parameters are obtained from the continuous infusion of inulin. The interassay $\mathrm{CV}$ for the inulin concentration were 6.9 and $1.9 \%$ at 136 and $725 \mathrm{mg} / \mathrm{l}$, respectively. An iGFR of $>120 \mathrm{ml} / \mathrm{min} / 1.73 \mathrm{~m}^{2}$ was set at $120 \mathrm{ml} / \mathrm{min} / 1.73 \mathrm{~m}^{2}$.

\section{Statistical analysis}

Patient data were collected from our hospital data storage unit where patient laboratory results are stored. Statistical analyses were performed with SPSS statistical software (version 22.0; 
IBM Corp., Armonk, NY) and Analyse-It for Excel ver. 3.91 (Analyse-It Software, Leeds, UK; Microsoft Corp., Redmond, WA). The eGFR calculated with the different equations were compared to the GFR calculated by inulin clearance (iGFR). A repeated-measures analysis of variance (ANOVA) was used to establish whether sequential measurements could serve as individual data points. We used Bland-Altman difference plot analysis to analyze agreement between iGFR and the eGFR determined using the different eGFR equations. This analysis results in a correlation between the two methods and limits of agreement, comprised of systemic (bias) and random (precision) error, where the limits of agreement can be used as a measure of total error [25]. Accuracy was calculated as follows: the difference between the iGFR and each of the eight eGFR outcomes was expressed as a percentage of the iGFR. To compare equations, we calculated the percentage of the samples whose eGFR differed by $\leq 10 \%( \pm 10 \%), \leq 20 \%$ $( \pm 20 \%)$, or $\leq 30 \%( \pm 30 \%)$, resulting in an accuracy of 90 , 80 , or $70 \%$, respectively.

We also compared CKD staging based on the different eGFR equations with that based on the iGFR. The National Kidney Foundation Kidney Disease Outcomes Quality Initiative (KDOQI) stages were applied, including the discrimination between stage $3 a$ and $3 b$, since the difference between these stages can have consequences for treatment and monitoring [26]. These stages are: stage 1, GFR $>90 \mathrm{ml} /$ $\mathrm{min} / 1.73 \mathrm{~m}^{2}$; stage $2,60-89 \mathrm{ml} / \mathrm{min} / 1.73 \mathrm{~m}^{2}$; stage $3 \mathrm{a}, 45-$ $59 \mathrm{ml} / \mathrm{min} / 1.73 \mathrm{~m}^{2}$ stage $3 \mathrm{~b}, 30-44 \mathrm{ml} / \mathrm{min} / 1.73 \mathrm{~m}^{2}$; stage 4 , $15-29 \mathrm{ml} / \mathrm{min} / 1.73 \mathrm{~m}^{2}$; stage $5,<15 \mathrm{ml} / \mathrm{min} / 1.73 \mathrm{~m}^{2}$. The abilities of the different eGFR equations to assign the correct
CKD class were compared with a McNemar's test, which is effectively a chi-square test on a $6 \times 6$ contingency table, and the Cohen's Kappa ( $\kappa)$ was calculated as a measure of agreement [27]. The $k$ coefficient indicates the proportion of agreement over and above chance agreement, with a $k$ value of 1 indicating complete agreement and a $\kappa$ value of 0 indicating no agreement. The $P$ value indicates whether the coefficient is statistically significantly different from zero.

\section{Results}

\section{Study subjects}

From February 2009 up to January 2013, 90 inulin clearance tests (iGFR range $13-178 \mathrm{ml} / \mathrm{min} / 1.73 \mathrm{~m}^{2}$ ) had been performed in 61 patients (age range 3.2-19.1 years). Forty-five and eight patients had undergone renal transplantation (70 measurements) and heart transplantation (12 measurements), respectively, two were pre-heart transplantation patients (2 measurements), and six patients (6 measurements) had a history of a malignancy (3 rhabdomyosarcoma, 2 Ewing sarcoma, 1 hepatoblastoma). Since our dataset contained sequential measurements we performed a repeated measures ANOVA analysis to test whether repeated measures would influence the outcome of the analyses. The interactions between the repeated measurements and eGFR outcome were not significant $(P=0.281)$. Descriptive characteristics of the study cohort are given in Table 1.
Table 1 Patient characteristics at time of inulin glomerular filtration rate measurement

\begin{tabular}{ll}
\hline Descriptive characteristics of patient cohort & Values \\
\hline Number of samples & 90 \\
Number of patients & 61 \\
Gender distribution of samples & Male $53(59 \%)$ \\
Age (years) & $12.5(7.8-16.4)$ \\
Weight (kg) & $39.7(18.5)$ \\
Height $(\mathrm{cm})$ & $141.5(23.3)$ \\
Post kidney transplant/post heart transplant/other & NTX $70(78 \%) /$ HTX $12(13 \%) /$ other $8(9 \%)$ \\
Inulin GFR range $\left(\mathrm{ml} / \mathrm{min} / 1.73 \mathrm{~m}^{2}\right)$ & $13-120$ \\
Inulin GFR median $\left(\mathrm{ml} / \mathrm{min} / 1.73 \mathrm{~m}^{2}\right)$ & $74(53-97)$ \\
CKD stage $($ number of measurements) & \\
CKD stage $5\left(<15 \mathrm{ml} / \mathrm{min} / 1.73 \mathrm{~m}^{2}\right)$ & $1(1 \%)$ \\
CKD stage $4\left(15-29 \mathrm{ml} / \mathrm{min} / 1.73 \mathrm{~m}^{2}\right)$ & $9(10 \%)$ \\
CKD stage $3 \mathrm{~b}\left(30-44 \mathrm{ml} / \mathrm{min} / 1.73 \mathrm{~m}^{2}\right)$ & $12(13 \%)$ \\
CKD stage $3 \mathrm{a}\left(45-59 \mathrm{ml} / \mathrm{min} / 1.73 \mathrm{~m}^{2}\right)$ & $23(26 \%)$ \\
CKD stage $2\left(60-89 \mathrm{ml} / \mathrm{min} / 1.73 \mathrm{~m}^{2}\right)$ & $21(23 \%)$ \\
CKD stage $1\left(>0 \mathrm{ml} / \mathrm{min} / 1.73 \mathrm{~m}^{2}\right)$ & $24(27 \%)$ \\
\hline
\end{tabular}

Data are presented as a number $(n)$ with/without the percentage in parenthesis, as appropriate, or as the median with the interquartile range (IQR) given in parenthesis

NTX, Post kidney transplant; HTX, post heart transplant; GFR, glomerular filtration rate; CKD, chronic kidney disease 


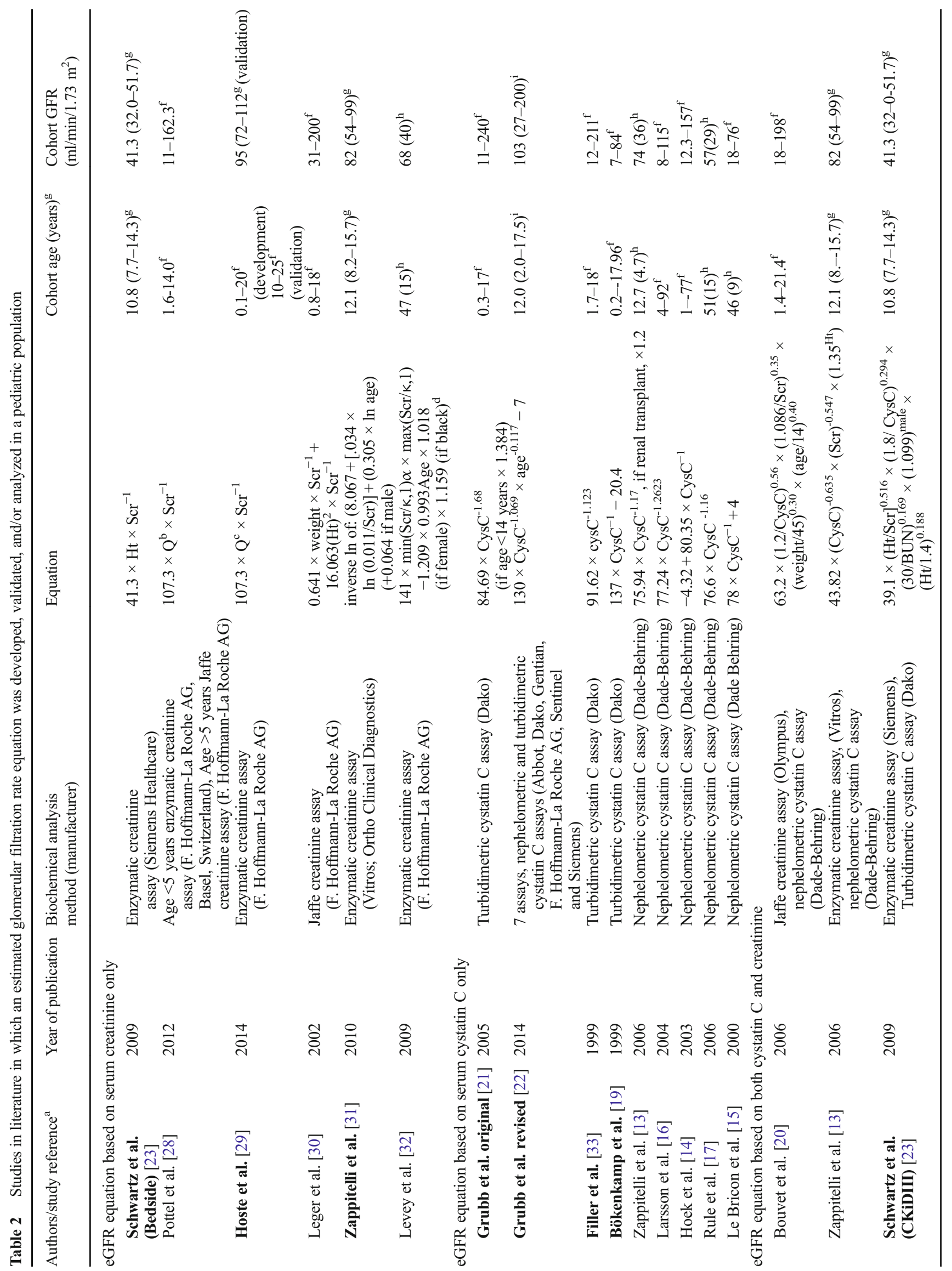




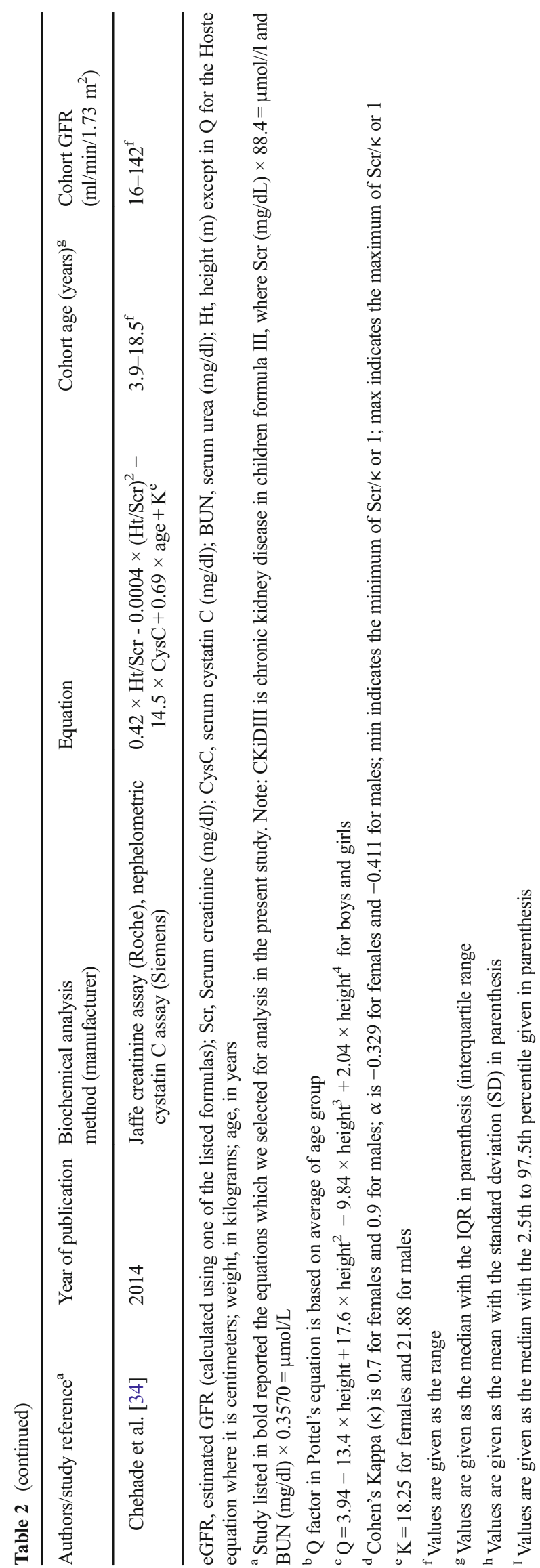

\section{Selection of eGFR equation}

Our literature search identified 20 cystatin C- and/or creatinine-based GFR formulas that had been developed or validated in children (Table 2). Of these 20 studies, seven (with 8 eGFR equations) met our criteria of comparable biochemical analysis, cohort age, and GFR distribution: Schwartz et al. (modified bedside and CKIDIII) [23], Zappitelli et al. [31], Hoste et al. [29], Grubb et al. (original and revised) [21, 22], Filler et al. [33], and Bökenkamp et al. [19]. The Schwartz modified bedside, Hoste, and Zappitelli equations are creatinine based, the Grubb original and revised, Filler, and Bökenkamp equations are cystatin $\mathrm{C}$ based, and the Schwartz CKiDIII is both creatinine and cystatin $\mathrm{C}$ based.

\section{eGFR equation performance}

The Bland-Altman difference plot analysis of the iGFR and the eGFRs from the eight equations is shown in Fig. 1, and the bias and limit of agreement values are presented in Table 3 . The mean bias of the tested equations ranged from -3.4 to 20.7, with the Hoste [29] equation showing the lowest bias. The original and revised Grubb [21, 22], Hoste [29], and CKiDIII Schwartz [23] equations did not show a significant bias (the $95 \%$ confidence interval of the bias did contain zero) in contrast to the other equations. The smallest interval for limits of agreement, and thereby the lowest total error, was shown by the CKiDIII Schwartz equation [23], followed by the Filler [33] and revised Grubb [22] equations. When we analyzed the performance of the equations using only the data from the renal transplant recipients, we saw a decrease in mean bias, a narrowing of the limits of agreement, and an increase in the coefficient of determination (Table 4).

The difference fit analysis showed that all equations resulted in an overestimation of GFR at low iGFR values and an underestimation of GFR at high iGFR values. Although overestimation is significant in all equations, the effect size varied from 27 (CKiDIII Schwartz [23]) to $47 \mathrm{ml} / \mathrm{min} / 1.73 \mathrm{~m}^{2}$ (Filler [33]). All equations showed a negative association between the iGFR and the difference between eGFR and iGFR. The CKiDIII Schwartz [23] equation resulted in the smallest interval for limits of agreement and the highest percentage of samples within 70, 80, and $90 \%$ accuracy (Table 5).

\section{Clinical stratification by CKD class}

Figure 2 shows per iGFR-CKD class the percentage of samples that were assigned to a certain CKD class based on the eGFR. Overall, the eGFR equations resulted in underestimation of CKD stage (overestimation of renal function) compared with the iGFR for patients with decreased iGFR, while the opposite was true for patients with normal GFR. In the group of iGFR-CKD1, the equation of Bökenkamp 

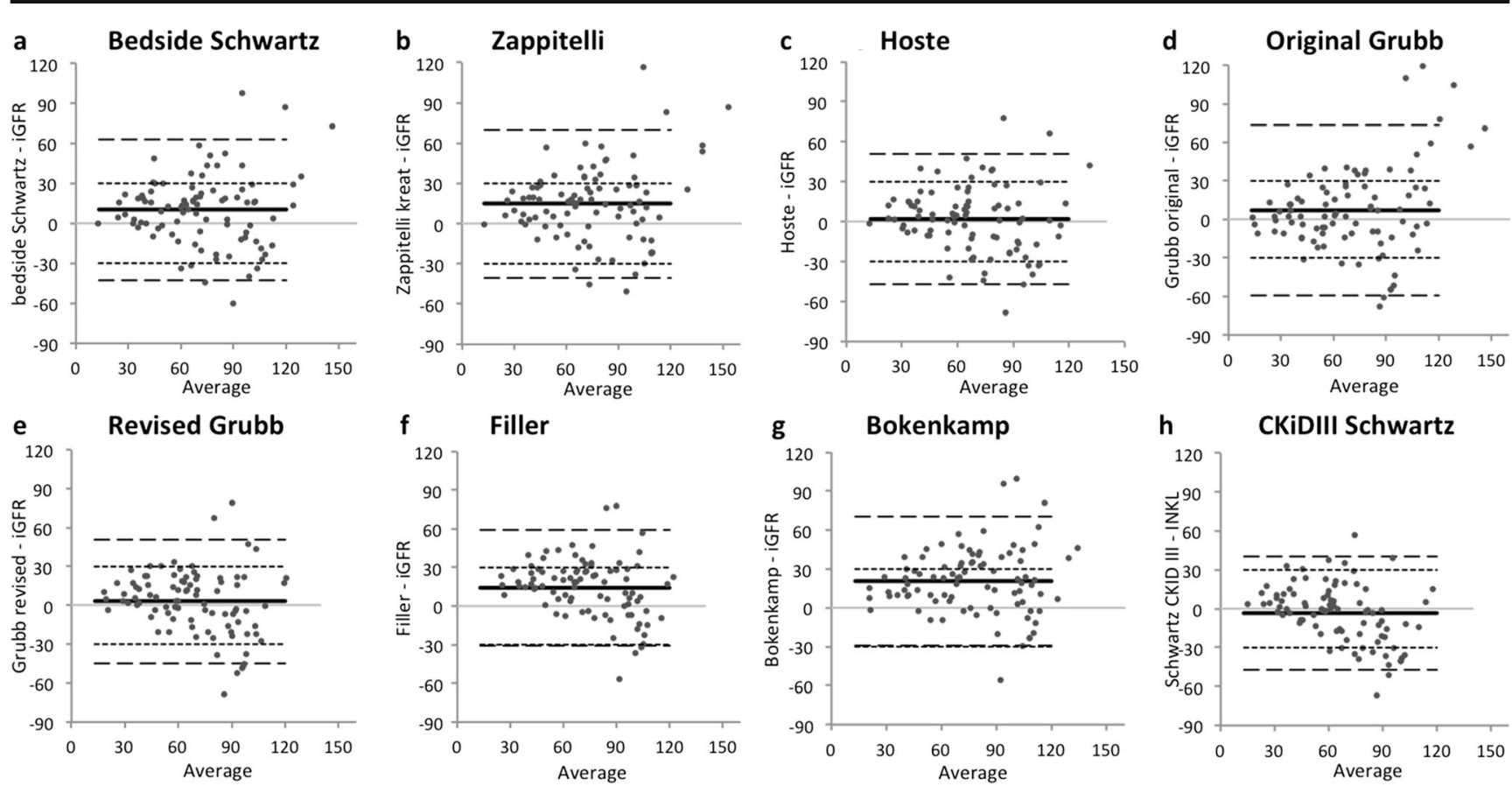

Fig. 1 Bland-Altman difference plot analysis between glomerular filtration rate determined by the inulin method $(i G F R)$ and estimated GFR (eGFR) as calculated using different equations. GFR is expressed in units of $\mathrm{ml} / \mathrm{min} / 1.73 \mathrm{~m}^{2}$. Horizontal axis Average iGFR and respective

eGFR values, solid line mean bias, broken lines $95 \%$ limits of agreement, dotted lines $\mathrm{p} 30$ (line indicating $30 \%$ difference between measurements and average). For chronic kidney disease (CKD) classification, see text (Statistical analysis section) and Table 1

To test whether classification by the eGFR equation difperformed best by correctly classifying 20 of 24 patients, whereas the CKiDIII Schwartz equation correctly classified only four of the 24 patients. The CKiDIII Schwartz equation correctly classified 12 of 20 patients with iGFR-CKD2, but seven patients were staged in a higher (worse) class. In the iGFR-CKD3a and -CKD3b classes, the CKiDIII Schwartz equation resulted in the highest number of correctly classified patients, and in the iGFR-CKD4 group, the Hoste equation resulted in the highest number of correctly classified patients ( 3 of 9 patients). No results are shown for the iGFR-CKD5 class as only one patient fell into this category.

fered from that by iGFR we used a McNemar chi-square test. All $P$ values were $<0.0001$, showing that the eGFR determined by all of the equations assessed were significantly different from the iGFR. To test the agreement between the classifications we performed a Cohen's $K$ analysis, the results of which are presented in Table 6. This test showed that agreement overall was poor to moderate. The highest $K$ value was observed for the bedside Schwartz equation (0.255), suggesting only moderate agreement at best with the CKD classification based on iGFR.

Table 3 Bias and limits of agreement values of Bland-Altman difference plot analysis ${ }^{\mathrm{a}}$ between glomerular filtration rate (GFR) determined by the inulin method and the estimated GFR calculated using the different equations

\begin{tabular}{lllll}
\hline eGFR equations & Bias (95\% CI) & Lower LoA $(95 \%$ CI $)$ & Upper LoA (95\% CI) & $\begin{array}{l}\text { Coefficient of } \\
\text { determination } R^{2}\end{array}$ \\
\hline Bedside Schwartz [23] & $10.0(4.4-15.7)$ & $-42.8(-52.5$ to -33.2$)$ & $62.9(53.2-72.5)$ & 0.614 \\
Zappitelli [31] & $14.6(8.7-20.6)$ & $-40.6(-50.8$ to -30.5$)$ & $69.9(59.8-80.0)$ & 0.615 \\
Hoste [29] & $1.9(-3.4$ to 7.1$)$ & $-47.1(-56.1$ to -38.1$)$ & $50.9(41.9-59.9)$ & 0.622 \\
Original Grubb [21] & $7.0(-0.1$ to 14.1$)$ & $-59.4(-71.6$ to -47.3$)$ & $73.4(61.2-85.6)$ & 0.538 \\
Revised Grubb [22] & $3.0(-2.1$ to 8.1$)$ & $-44.9(-53.7$ to -36.1$)$ & $50.8(42.1-59.6)$ & 0.619 \\
Filler [33] & $14.2(9.4-19.0)$ & $-30.8(-39.0$ to -22.5$)$ & $59.2(51.0-67.5)$ & 0.655 \\
Bökenkamp [19] & $20.7(15.4-26.0)$ & $-29.2(-38.3$ to -20.0$)$ & $70.6(61.4-79.7)$ & 0.655 \\
CKiDIII Schwartz [23] & $-3.4(-8.1$ to 1.3$)$ & $-47.2(-55.3$ to -39.1$)$ & $40.5(32.4-48.5)$ & 0.671 \\
\hline
\end{tabular}

LoA, limits of agreement; CI, confidence interval; iGFR, inulin-based GFR

${ }^{a}$ Analysis on whole dataset, including renal, and heart transplant recipients, and oncology patients (61 patients, 90 measurements). Graphical representation is shown in Fig. 1 
Table 4 Bias and limits of agreement values of Bland-Altman difference plot analysis ${ }^{\mathrm{a}}$ between iGFR and eGFR calculated using different equations

\begin{tabular}{lllll}
\hline eGFR equations & Bias $(95 \%$ CI $)$ & Lower LoA $(95 \%$ CI $)$ & Upper LoA $(95 \%$ CI) & Coefficient of determination $R^{2}$ \\
\hline Bedside Schwartz [23] & $6.8(1.1-12.5)$ & $-39.8(-49.6$ to -30.1$)$ & $53.4(43.7-63.2)$ & 0.678 \\
Zappitelli [31] & $11.7(5.8-17.6)$ & $-36.9(-47.0$ to -26.7$)$ & $60.3((50.1-70.4)$ & 0.683 \\
Hoste [29] & $-0.8(-6.22$ to 4.6$)$ & $-45.4(-54.8$ to -36.1$)$ & $43.9(34.5-53.2)$ & 0.675 \\
Original Grubb [21] & $2.4(-3.8$ to 8.5$)$ & $-48.2(-58.8$ to -37.7$)$ & $52.9(42.4-63.5)$ & 0.668 \\
Revised Grubb [22] & $1.0(-3.9$ to 5.9) & $-39.5(-47.9$ to -31.0$)$ & $41.4(33.0-49.9)$ & 0.727 \\
Filler [33] & $12.3(7.6-17.0)$ & $-26.4(-34.5$ to -18.3$)$ & $51.0(42.9-59.0)$ & 0.756 \\
Bökenkamp [19] & $17.0(12.1-21.8)$ & $-23.1(-31.5$ to 14.7$)$ & $57.0(48.7-65.4)$ & 0.756 \\
CKiDIII Schwartz [23] & $-4.6(-9.5$ to 0.4$)$ & $-45.3(-53.8$ to -36.8$)$ & $36.2(27.7-44.7)$ & 0.724 \\
\hline
\end{tabular}

$i G F R$ inulin-based glomerular filtration rate, $e G F R$ estimated glomerular filtration rate

${ }^{a}$ Analysis on data obtained only from renal transplant recipients (45 patients, 70 measurements)

\section{Discussion}

In this study we addressed the efficacy of eight different equations to estimate GFR based on serum concentrations of creatinine and/or cystatin C compared to the GFR based on inulin clearance in a pediatric cohort of 61 children who were followed after kidney or heart transplantation or after nephrotoxic chemotherapy. Even though the selected GFR equations were matched to our study cohort in terms of age, the GFR range, and the biochemical methods used, the results varied widely, and none of the equations performed particularly well. Even the best coefficient of determination was only $R^{2}=0.67$, which is very modest given that eGFR and iGFR are supposed to be measuring the same thing and, therefore, are expected to be highly correlated.

Based on the limits of agreement, the CKiDIII Schwartz [23] equation performed best with a LoA interval of 87.8. This equation also showed the highest accuracy with 26,44 , and $64 \%$ of samples differing by $<10,<20$, or $<30 \%$ from the reference method (iGFR), respectively, followed by the Hoste and revised Grubb equations.

With regard to bias the equation by Hoste et al. [29] showed the lowest bias (1.9), followed by the revised
Grubb, CKiDIII, and original Grubb equations, with bias of $3.0,-3.4$, and 7.0, respectively, all of which were not significant.

Nehus et al. [35] and Bacchetta et al. [36] showed a much better performance of the eGFR equations they investigated in their respective studies, with up to 44 and $48 \%$ of the samples within $90 \%$ accuracy, respectively. This difference from our results may be due to differences in the iGFR range: in the studies of Bacchetta et al. [36] and Nehus et al. [35], the normal range of the iGFR was $100 \pm 32 \mathrm{ml} / \mathrm{min} / 1.73 \mathrm{~m}^{2}$ [mean \pm standard deviation (SD)] and $95(76-110) \mathrm{ml} / \mathrm{min} /$ $1.73 \mathrm{~m}^{2}$ [median (interquartile range)]. Both values are considerably higher than the iGFR in our cohort [69 (52-94) $\mathrm{ml} /$ $\left.\min / 1.73 \mathrm{~m}^{2}\right]$ [mean $\left.( \pm \mathrm{SD})\right]$. The inclusion of adolescents in our cohort, in contrast to the CKiDIII cohort, may have decreased the performance of the CKiDIII Schwartz, which in the study of Nehus et al. [35] did perform better than in our study. Therefore, the difference in renal function between the study cohorts in these studies and our cohort likely explains the difference in performance.

An interesting finding of our study is that those equations which performed best analytically (CKiDIII, Hoste, and revised Grubb equations) were not the equations which

Table 5 Accuracy analysis

\begin{tabular}{llcc}
\hline eGFR equations & $70 \%$ accuracy & $80 \%$ accuracy & $90 \%$ accuracy \\
\hline Bedside Schwartz [23] & $59 \%$ & $34 \%$ & $19 \%$ \\
Zappitelli [31] & $52 \%$ & $33 \%$ & $16 \%$ \\
Hoste [29] & $61 \%$ & $46 \%$ & $21 \%$ \\
Original Grubb [21] & $52 \%$ & $38 \%$ & $20 \%$ \\
Revised Grubb [22] & $61 \%$ & $44 \%$ & $21 \%$ \\
Filler [33] & $43 \%$ & $34 \%$ & $18 \%$ \\
Bökenkamp [19] & $42 \%$ & $33 \%$ & $19 \%$ \\
CKIDIII Schwartz [23] & $64 \%$ & $44 \%$ & $26 \%$
\end{tabular}

Values in table are the percentages of estimated glomerular filtration rate (eGFR) results per equation that differ by $<30,<20$, or $<10 \%$ from the inulinbased glomerular filtration rate (iGFR), resulting in 70,80 , or $90 \%$ accuracy, respectively 


\section{CKD1}

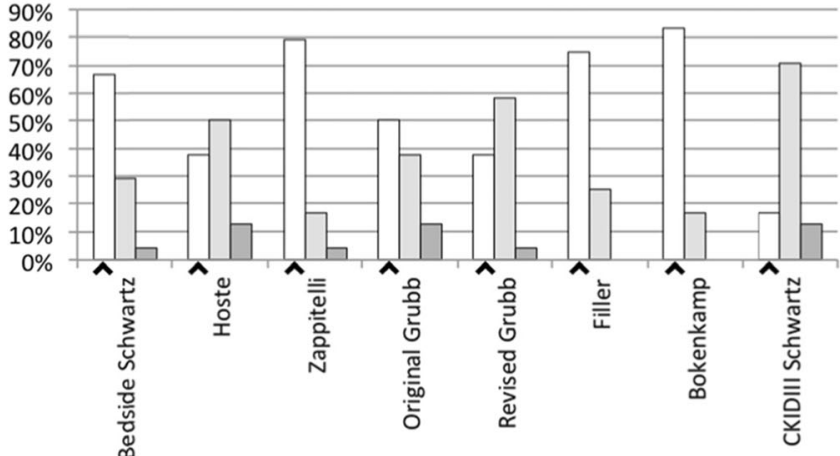

CKD3a

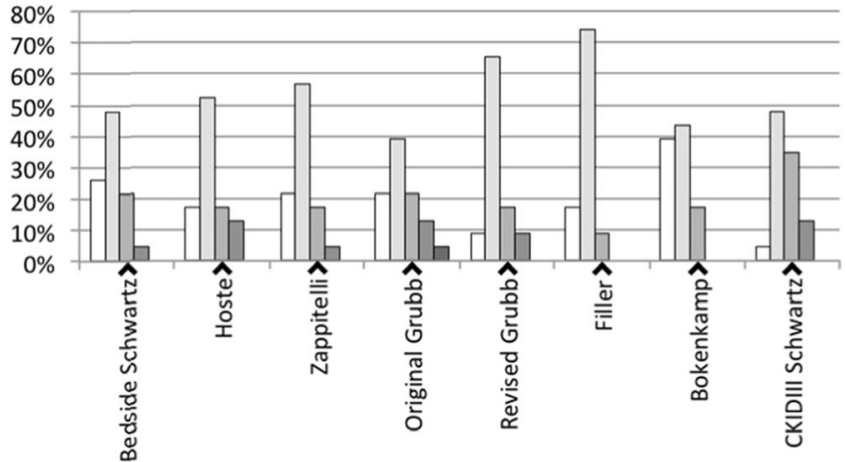

CKD4

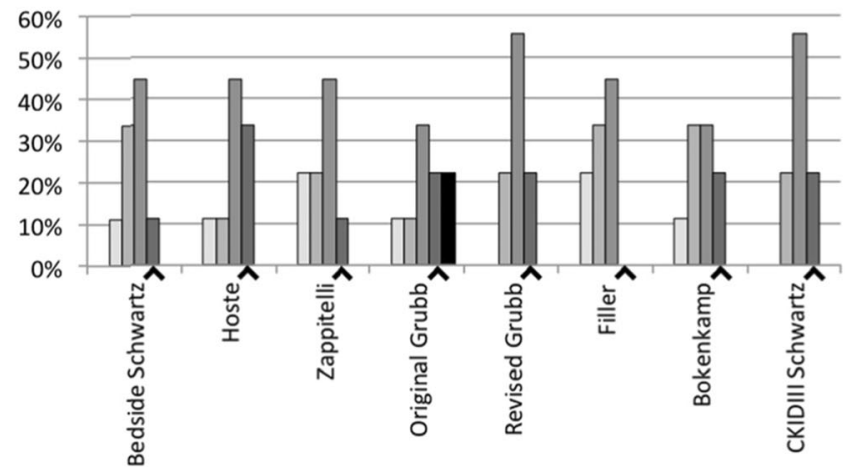

Fig. 2 Chronic kidney disease (CKD) classification of patients according to estimated glomerular filtration rate (eGFR) calculated using the different equations, sorted by inulin-based glomerular filtration rate

performed best in terms of CKD classification. This can be explained by the unequal distribution of the number of patients over the different CKD classes. When only patients in CKD classes $3 \mathrm{a}, 3 \mathrm{~b}$, and 4 were analyzed, the CKiDIII, Hoste, and revised Grubb equations successively had the highest number of correctly classified patients.

In our study, we saw that there was a trend for all equations, both cystatin $\mathrm{C}$ - and/or creatinine-based, toward overestimating renal function at lower iGFR values and underestimating it at higher iGFR values. This trend was also reflected in CKD staging: when we used the eGFR to assign a CKD stage to every patient we observed an overall tendency

\section{CKD2}

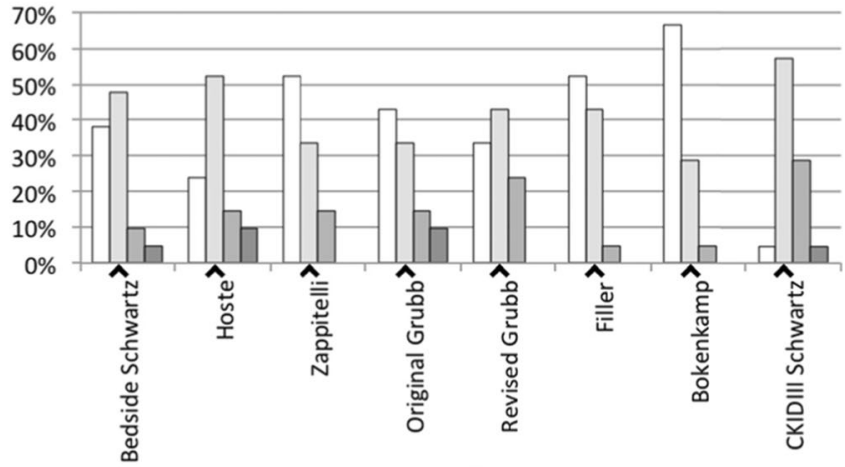

CKD3b

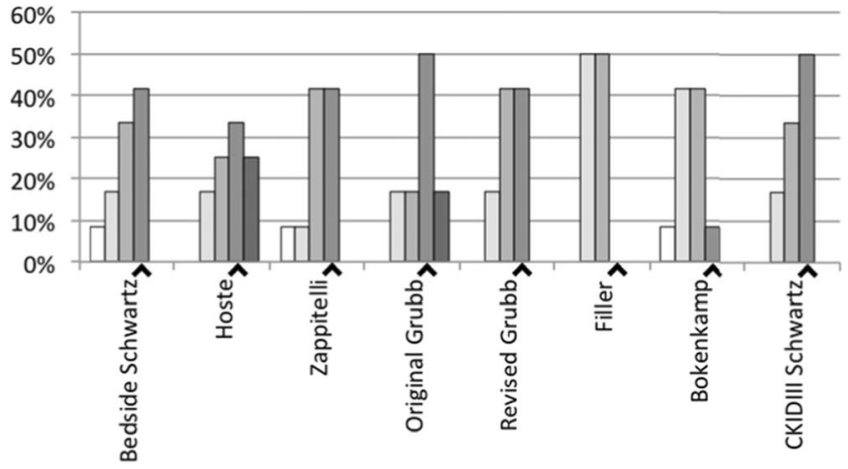

$\square$ ckd1

$\square$ ckd2

$\square_{\text {ckd3a }}$

$\square \mathrm{ckd} 3 \mathrm{~b}$

$\square$ ckd4

- $\mathrm{ckd5}$

(iGFR)-CKD class. Black arrowheads highlights the correct eGFR class for the iGFR. GFR is expressed in units of $\mathrm{ml} / \mathrm{min} / 1.73 \mathrm{~m}^{2}$. For CKD classification, see text (Statistical analysis section) and Table 1

to stage patients at a lower (better) class based on their eGFR than on their iGFR. In contrast, in patients with a higher iGFR, renal function will be underestimated, although this will not affect CKD staging, as for most equations this point is $\geq 90$. In general practice, this overestimation of renal function and incorrect CKD classification can cause delay in treatment, overdosage of medication, and/or inadequate follow up.

The mathematical manipulations in the eGFR equations assessed could potentially explain the difference in performance between them. In the equations based only on cystatin $\mathrm{C}$, the result of the cystatin $\mathrm{C}$ assay had a stronger effect on the outcome of the original Grubb equation [21] than on that of 
Table 6 Summary of chronic kidney disease category analysis

\begin{tabular}{lll}
\hline eGFR equations & Percentage correctly classified samples & Cohen's kappa (K) $(95 \% \mathrm{CI})^{\mathrm{a}}$ \\
\hline Bedside Schwartz [23] & 41.6 & $0.255(0.126-0.384)$ \\
Zappitelli [31] & 40.4 & $0.230(0.103-0.357)$ \\
Hoste [29] & 34.8 & $0.176(0.049-0.303)$ \\
Grubb original [21] & 36.0 & $0.177(0.052-0.302)$ \\
Grubb revised [22] & 32.6 & $0.124(-0.003$ to 0.251$)$ \\
Filler [33] & 32.6 & $0.105(-0.005$ to 0.215$)$ \\
Bokenkamp [19] & 37.1 & $0.162(0.046-0.278)$ \\
CKIDIII Schwartz [23] & 36.0 & $0.152(0.036-0.268)$ \\
\hline
\end{tabular}

Data are presented as the percentage of correctly classified samples in classes iGFR-CKD1 through to IGFR-CKD4 of all 89 samples in these groups $C K D$ chronic kidney disease, $i G F R$ inulin-based glomerular filtration rate, $e G F R$ estimated glomerular filtration rate

${ }^{a}$ Cohen's kappa ( $\mathrm{K}$ ) analysis of CKD was used to categorize eGFR results (CKD-eGFR) compared to the iGFR (CKD-iGFR)

the Filler equation [33]. In the revised Grubb equation [22], age has become an exponential factor, whereas in the original Grubb equation it was a dichotomous factor; this change possibly results in a better correlation to the iGFR. The CKiDIII Schwartz equation [23] is very complex because apart from including the height/creatinine ratio, serum cystatin $\mathrm{C}$ level, and blood urea level, it also has height as a separate exponential factor and gender as a factor. By including so many parameters that influence the outcome of this equation, each individual parameter has a smaller effect on the eGFR calculated using this formula than in a simpler equation. As a result, such the CKiDIII Swartz equation would be more resistant to aberrations in the parameters on an individual patient level, which can explain why in our study it outperformed the other equations. On the other hand, the simplicity of the bedside Schwartz equation makes it a useful tool in daily practice.

The study by Hoste et al. [29] shows the importance of height correction in eGFR, as was also highlighted by Schwartz [37] and confirmed in studies by Rink et al. [38] and de Souza et al. [39]. All of these studies show that height correction is particularly relevant in adolescent patients. Our study cohort included adolescents, but we did not analyze specific adolescent and pre-adolescent equations. In these mixed populations the use of height-dependent equations can have additional value. Of the equations included in our study, only the Schwartz bedside equation, the CKiDIII equation, and the Hoste equation use height in the calculation of the eGFR.

The equations analyzed in this study were developed in studies with patient cohorts with suspected or established renal pathology. The cohort in our study also included patients before and after heart transplantation or in their follow-up after chemotherapy. The inclusion of a variety of patients may influence the outcome of the performance analysis. Comparing the performance of the equations in these subgroups to that of the whole study cohort would give more insight into the use of these equations in specific patient groups. However, the subgroups of oncology and heart transplantation were very small in our study, making statistical analysis unfeasible. Analysis of only the renal transplant recipient data showed some improvement in performance for all equations. Consequently, using these equations in patients suffering from renal pathology will yield more reliable results than in other patient groups.

Although much work has been done on pediatric equations using creatinine and cystatin $\mathrm{C}$ as biomarkers, we found that in our cohort the performance of such equations was modest at best. Better methods, or better equations, are needed to improve and harmonize this field.

Our study has two major strengths. First, we selected eGFR equations from the literature that had been validated in a cohort comparable to our cohort in terms of age, GFR range, and biochemical methods used. This ensured that only eGFR equations were included that should perform best in our cohort. Second, we related the calculated eGFR to CKD staging, which provided good insight in the clinical consequences of implementing these eGFR equations in daily practice. To our knowledge, this latter analysis has not been done previously for pediatric equations.

One limitation needs to be addressed. We did not use the new International Federation of Clinical Chemistry and Laboratory Medicine (IFCC)-calibrated cystatin C assay. The IFCC reference material was established in 2010. Until recently, almost all studies in the literature have used a nonIFCC calibrated cystatin $C$ assay, as a result of which the cystatin $\mathrm{C}$ results can be biased, which will affect the eGFR equation that was developed. This bias can lead to inaccuracy in some equations. An exception is a recently published multicenter study by Grubb et al. [22] in which different IFCC standardized assays were used to determine a new (revised) equation to calculate the eGFR from the cystatin $\mathrm{C}$ concentration in blood. The use of an IFCC calibrated Roche assay will result in concentrations that are approximately $15 \%$ lower than those used in our earlier assays (internal communication with Roche Diagnostics). As a result, the eGFR from the Grubb 2014 equation will be $23 \%$ higher. When we corrected 
for the $15 \%$ difference between our assay and the new IFCCcalibrated assay we found a mean bias of 22.6. A recent study by Eckfeldt et al. did not show a $15 \%$ decrease, but rather a $3.3 \%$ increase in cystatin $\mathrm{C}$ results when using the Roche IFCC-calibrated assay, which corresponds with our findings using the revised Grubb equation [40].

In conclusion, in our cohort of pediatric and adolescent patients we found the CKiDIII equation to be the best alternative to the inulin clearance test, closely followed by the Hoste and revised Grubb equations, although the performance of all three equations was only moderate at best. All eight equations assessed showed inconsistency throughout the range of iGFR, overestimating renal function at low iGFR values and underestimating renal function at high ones. Since for daily monitoring of renal function and screening of patients most equations may be too elaborate, the modified bedside Schwartz equation remains an acceptable alternative to the iGFR.

Acknowledgments We would like to thank S P Willemsen, MSc, of the Department of Biostatistics of the Erasmus MC University Medical Center, Rotterdam, for his contribution and input on statistical analysis and Drs J. Hagoort for English language editing of the manuscript.

Compliance with ethical standards The Medical Ethical Review Board of the Erasmus Medical Center waived the necessity of informed consent considering that the subjects are not submitted to any additional handling.

Conflict of interest The authors declare that they have no conflict of interest

Open Access This article is distributed under the terms of the Creative Commons Attribution 4.0 International License (http:// creativecommons.org/licenses/by/4.0/), which permits unrestricted use, distribution, and reproduction in any medium, provided you give appropriate credit to the original author(s) and the source, provide a link to the Creative Commons license, and indicate if changes were made.

\section{References}

1. Brandstrom E, Grzegorczyk A, Jacobsson L, Friberg P, Lindahl A, Aurell M (1998) GFR measurement with iohexol and ${ }^{51} \mathrm{Cr}$-EDTA. A comparison of the two favoured GFR markers in Europe. Nephrol Dial Transplant 13:1176-1182

2. Florkowski CM, Chew-Harris JS (2011) Methods of estimating GFR - different equations including CKD-EPI. Clin Biochem Rev 32:75-79

3. Stevens LA, Coresh J, Greene T, Levey AS (2006) Assessing kidney function - measured and estimated glomerular filtration rate. $\mathrm{N}$ Engl J Med 354:2473-2483

4. Levey AS (1990) Measurement of renal function in chronic renal disease. Kidney Int 38:167-184

5. Perrone RD, Madias NE, Levey AS (1992) Serum creatinine as an index of renal function: new insights into old concepts. Clin Chem 38:1933-1953
6. Levey AS, Perrone RD, Madias NE (1988) Serum creatinine and renal function. Annu Rev Med 39:465-490

7. Shemesh O, Golbetz H, Kriss JP, Myers BD (1985) Limitations of creatinine as a filtration marker in glomerulopathic patients. Kidney Int 28:830-838

8. Schwartz GJ, Haycock GB, Edelmann CMJ, Spitzer A (1976) A simple estimate of glomerular filtration rate in children derived from body length and plasma creatinine. Pediatrics 58:259-263

9. Grubb A, Simonsen O, Sturfelt G, Truedsson L, Thysell H (1985) Serum concentration of cystatin C, factor D and beta 2microglobulin as a measure of glomerular filtration rate. Acta Med Scand 218:499-503

10. Filler G, Bokenkamp A, Hofmann W, Le Bricon T, Martinez-Bru GA (2005) Cystatin C as a marker of GFR - history, indications, and future research. Clin Biochem 38:1-8

11. Andersen TB, Eskild-Jensen A, Frokiaer J, Brochner-Mortensen J (2009) Measuring glomerular filtration rate in children; can cystatin C replace established methods? A review. Pediatr Nephrol 24:929 941

12. Sjostrom P, Tidman M, Jones I (2005) Determination of the production rate and non-renal clearance of cystatin $\mathrm{C}$ and estimation of the glomerular filtration rate from the serum concentration of cystatin C in humans. Scand J Clin Lab Invest 65:111-124

13. Zappitelli M, Parvex P, Joseph L, Paradis G, Grey V, Lau S, Bell L (2006) Derivation and validation of cystatin C-based prediction equations for GFR in children. Am J Kidney Dis 48:221-230

14. Hoek FJ, Kemperman FA, Krediet RT (2003) A comparison between cystatin C, plasma creatinine and the Cockcroft and Gault formula for the estimation of glomerular filtration rate. Nephrol Dial Transplant 18:2024-2031

15. Le Bricon T, Thervet E, Froissart M, Benlakehal M, Bousquet B, Legendre C, Erlich D (2000) Plasma cystatin C is superior to 24-h creatinine clearance and plasma creatinine for estimation of glomerular filtration rate 3 months after kidney transplantation. Clin Chem 46:1206-1207

16. Larsson A, Malm J, Grubb A, Hansson LO (2004) Calculation of glomerular filtration rate expressed in $\mathrm{mL} / \mathrm{min}$ from plasma cystatin $\mathrm{C}$ values in $\mathrm{mg} / \mathrm{L}$. Scand J Clin Lab Invest 64:25-30

17. Rule AD, Bergstralh EJ, Slezak JM, Bergert J, Larson TS (2006) Glomerular filtration rate estimated by cystatin $\mathrm{C}$ among different clinical presentations. Kidney Int 69:399-405

18. Filler G, Lepage N (2003) Should the Schwartz formula for estimation of GFR be replaced by cystatin C formula? Pediatr Nephrol 18: 981-985

19. Bokenkamp A, Domanetzki M, Zinck R, Schumann G, Byrd D, Brodehl J (1999) Cystatin C serum concentrations underestimate glomerular filtration rate in renal transplant recipients. Clin Chem 45:1866-1868

20. Bouvet Y, Bouissou F, Coulais Y, Séronie-Vivien S, Tafani M, Decramer S, Chatelut E (2006) GFR is better estimated by considering both serum cystatin $\mathrm{C}$ and creatinine levels. Pediatr Nephrol 21:1299-1306

21. Grubb A, Nyman U, Bjork J, Lindström V, Rippe B, Sterner G, Christensson A (2005) Simple cystatin C-based prediction equations for glomerular filtration rate compared with the modification of diet in renal disease prediction equation for adults and the Schwartz and the Counahan-Barratt prediction equations for children. Clin Chem 51:1420-1431

22. Grubb A, Horio M, Hansson LO, Björk J, Nyman U, Flodin M, Larsson A, Bökenkamp A, Yasuda Y, Blufpand H, Lindström V, Zegers I, Althaus H, Blirup-Jensen S, Itoh Y, Sjöström P, Nordin G, Christensson A, Klima H, Sunde K, Hjort-Christensen P, Armbruster D, Ferrero C (2014) Generation of a new cystatin Cbased estimating equation for glomerular filtration rate by use of 7 assays standardized to the international calibrator. Clin Chem 60: 974-986 
23. Schwartz GJ, Munoz A, Schneider MF, Mak RH, Kaskel F, Warady BA, Furth SL (2009) New equations to estimate GFR in children with CKD. J Am Soc Nephrol 20:629-637

24. van Rossum LK, Cransberg K, de Rijke YB, Zietse R, Lindemans J, Vulto AG (2005) Determination of inulin clearance by single injection or infusion in children. Pediatr Nephrol 20:777-781

25. Bland JM, Altman DG (1986) Statistical methods for assessing agreement between two methods of clinical measurement. Lancet $1: 307-310$

26. Levey AS, Eckardt K-U, Tsukamoto Y, Levin A, Coresh J, Rossert J, de Zeeuw D, Hostetter TH, Lameire N, Eknoyan G (2005) Definition and classification of chronic kidney disease: a position statement from Kidney Disease: Improving Global Outcomes (KDIGO). Kidney Int 67:2089-2100

27. Landis JR, Koch GG (1977) The measurement of observer agreement for categorical data. Biometrics 33:159-174

28. Pottel H, Hoste L, Martens F (2012) A simple height-independent equation for estimating glomerular filtration rate in children. Pediatr Nephrol 27:973-979

29. Hoste L, Dubourg L, Selistre L, De Souza VC, Ranchin B, HadjAïssa A, Cochat P, Martens F, Pottel H (2014) A new equation to estimate the glomerular filtration rate in children, adolescents and young adults. Nephrol Dial Transplant 29:1082-1091

30. Léger F, Bouissou F, Coulais Y, Tafani M, Chatelut E (2002) Estimation of glomerular filtration rate in children. Pediatr Nephrol 17:903-907

31. Zappitelli M, Zhang X, Foster BJ (2010) Estimating glomerular filtration rate in children at serial follow-up when height is unknown. Clin J Am Soc Nephrol 5:1763-1769

32. Levey AS, Stevens LA, Schmid CH, Zhang Y, Castro AF III, Feldman HI, Kusek JW, Eggers P, Van Lente F, Greene T, Coresh
J (2009) A new equation to estimate glomerular filtration rate. Ann Intern Med 150:604-612

33. Filler G, Priem F, Vollmer I, Gellermann J, Jung K (1999) Diagnostic sensitivity of serum cystatin for impaired glomerular filtration rate. Pediatr Nephrol 13:501-505

34. Chehade H, Cachat F, Jannot AS, Meyrat BJ, Mosig D, Bardy D, Parvex P, Girardin E (2014) New combined serum creatinine and cystatin C quadratic formula for GFR assessment in children. Clin J Am Soc Nephrol 9:54-63

35. Nehus EJ, Laskin BL, Kathman TI, Bissler JJ (2013) Performance of cystatin C-based equations in a pediatric cohort at high risk of kidney injury. Pediatr Nephrol 28:453-461

36. Bacchetta J, Cochat P, Rognant N, Ranchin B, Hadj-Aissa A, Dubourg L (2011) Which creatinine and cystatin C equations can be reliably used in children? Clin J Am Soc Nephrol 6:552-560

37. Schwartz GJ (2014) Height: the missing link in estimating glomerular filtration rate in children and adolescents. Nephrol Dial Transplant 29:944-947

38. Rink N, Zappitelli M (2015) Estimation of glomerular filtration rate with and without height: effect of age and renal function level. Pediatr Nephrol 30:1327-1336

39. De Souza V, Pottel H, Hoste L, Dolomanova O, Cartier R, Selistre L, Ranchin B, Hadj-Aïssa A, Lemoine S, Cochat P, Dubourg L (2015) Can the height-independent Pottel eGFR equation be used as a screening tool for chronic kidney disease in children? Eur J Pediatr 174:1225-1235

40. Eckfeldt JH, Karger AB, Miller WG, Rynders GP, Inker LA (2015) Performance in measurement of serum cystatin $\mathrm{C}$ by Laboratories Participating in the College of American Pathologists 2014 CYS Survey. Arch Pathol Lab Med 139:888-893 